\title{
LA SUPERVISIÓN EDUCATIVA EN EL CONTEXTO DE LOS SISTEMAS EDUCATIVOS LATINOAMERICANOS
}

\author{
Ernesto Fajardo Pascagaza ${ }^{1}$
}

\begin{abstract}
Resumen: El presente texto tiene como finalidad realizar un análisis sobre la importancia de la supervisión en los procesos administrativos y pedagógicos, así como en los replanteamientos que los sistemas educativos en América Latina están haciendo para mejorar la calidad de la educación desde la administración escolar. Igualmente, se hace un análisis sobre los procesos que deben desarrollar los principales actores para mejorar la calidad y lograr la transformación de los centros educativos, donde el supervisor escolar representa una figura fundamental en el proceso, pero más determinante es la que desempeña el docente. En este sentido, se analizan las variables inherentes al rol que ejercen frente a conductas que vulneran los procesos y las prácticas de supervisión y gestión escolar y el rol que ejerce el supervisor como el principal gestor para mejorar la calidad de la educación en las instituciones educativas. Para finalizar, se realiza una aproximación a los modelos contemporáneos sobre la supervisión escolar así como el impacto que han tenido las reformas educativas en el proceso de transformación de los sistemas educativos en América Latina.
\end{abstract}

Palabras clave: Supervisión educativa, América Latina, Sistemas educativos.

\section{EDUCATIONAL SUPERVISION IN THE CONTEXT OF LATIN AMERICAN EDUCATIONAL SYSTEMS}

\begin{abstract}
Abstrac: The purpose of this text is to analyze the importance of supervision in administrative and pedagogical processes, as well as in the rethinking that educational systems in Latin America are doing to improve the quality of education from school administration. Likewise, an analysis is made of the processes that must be developed by the main actors to improve the quality and achieve the
\end{abstract}

1 Doctorando en Educación, Doctorando en Filosofía, Magíster en Filosofía y Magíster en Educación, Magister en Teología. Licenciado en Teología y Licenciado en Filosofía, Especialista en Filosofía y Educación, con Formación Sacerdotal. Docente del Departamento de Humanidades y Formación Integral de la USTA. Docente de la Universidad Militar. Integrante del Grupo de Investigación ALETHEIA. Investigador principal. Miembro del Observatorio de la Diversidad Religiosa y Cultural en América Latina y el Caribe (ODREC).Correo electrónico: ernestofajardo@usantotomas.edu.co Artículo producto de la investigación realizada al interior del Grupo de Investigación ALETHEIA de la Universidad Santo Tomás, en torno a la relación entre educación, ética y política 
transformation of the educational centers, where the school supervisor represents a fundamental figure in the process, but more determinant is that performed by the teacher. In this sense, we analyze the variables inherent to the role they play in the face of behaviors that violate the processes and practices of school supervision and management and the role played by the supervisor as the main manager to improve the quality of education in educational institutions. . Finally, an approach is made to contemporary models of school supervision and the impact that educational reforms have had on the process of transforming educational systems in Latin America.

Keywords: Educational supervision, Latin America, Educational systems.

\section{Introducción}

La supervisión origina cambios, reformas e innovaciones en los contextos, momentos, situaciones y sistemas educativos teniendo como referente su intervención con autoridad desde sus funciones y competencias establecidas. En este orden, la inspección como sinónimo de la supervisión informa, asesora, media, controla, evalúa, así como avala la calidad educativa. La supervisión, media entre la administración y el centro educativo para el correcto y ágil funcionamiento del control y evaluación en aras de la calidad educativa y esa es la tarea que se asume en Iberoamérica a partir de la asunción de las reformas educativas generadoras de cambios administrativos, respondiendo a las demandas y estructurales, respondiendo a los procesos y sistemas evaluativos internacionales y su impacto y adaptación en las políticas educativas nacionales.

Las pruebas han de ser direccionadas hacia la formación ciudadana fundamentada en el pensamiento crítico independiente y autónomo y por consiguiente implicando la toma de decisiones en la cotidianidad de las personas. En este sentido, la supervisión procura los cambios en las estructuras como eje articulador de la calidad en los sistemas educativos yendo de la mano de la dirección de los centros docentes para responder idóneamente a los requerimientos de la sociedad.

Uno de los objetivos que fundamentan y orientan la labor de la supervisión educativa es la de ayudar y asesorar a los docentes a que generen actitudes de liderazgo democrático, establezcan lazos morales con quienes pertenecen a los contextos de la comunidad educativa, así como a ponderar procesos evaluativos entorno a los logros obtenidos de acuerdo a los objetivos que se hayan propuesto y por consiguiente, respondiendo a las necesidades educativas.

Los Distritos Escolares son unidades básicas que tienen como competencia la supervisión de planteles, centros, comunidades educativas, formación docente, cátedras, así como servicios educativos. En este orden, tienen como funciones la de dirigir, coordinar, supervisar y evaluar la correspondiente ejecución de las políticas educativas de tal manera que ejecute programas tendientes a la supervisión del trabajo docente y la administración de los procesos que tiendan a mejorar la calidad de la enseñanza en los planteles educativos.

Es claro entonces que el sector educativo ejecuta la labor de supervisión, velando por el funcionamiento adecuado del plan operativo del sector, coordina 
actividades sociales y académicas, asesora y orienta a quien dirige el plantel para el correcto funcionamiento de programas, así como atiende a directivos, padres de familia y representantes en la resolución de problemas.

En cuanto a los enfoques de la labor supervisora, está el de inspección porque controla lo concerniente a la responsabilidad administrativa, el tecnológico porque ayuda a cambiar la realidad educativa, el clínico en tanto libera a la labor de supervisión de las prácticas que la afectan y el crítico, que complementa aquellos eventos omitidos y que denotan deficiencia en la supervisión. La labor de supervisión se articula con la calidad en la asunción de cambios profundos y transformadores tendientes a mejorar el ser, el hacer, el conocer y el convivir de todos los escenarios educativos.

Las escuelas están experimentando profundos cambios desde la supervisión educativa, tanto exógena como endógena y esto se evidencia en el accionar y la gestión de los supervisores. Según la OCDE (2004), desde la década de los noventas, no se han presentado innovadores resultados que respalden la inversión que se ha hecho a partir de la reforma educativa porque las escuelas no han mejorado sus prácticas y resultados, dado que se han acentuado diferencias entre las escuelas de tal manera que las escuelas que son más desfavorecidas con recursos, debido al sistema competitivo, quedan rezagadas y justo son las escuelas públicas que ocupan el $82 \%$ de la cobertura en educación pública nacional. Igualmente, la supervisión educativa no ha vinculado con fortaleza los objetivos y los esfuerzos desde las políticas educativas y su correspondencia con la praxis de las escuelas y en este orden, el consecuente control de la calidad educativa, que sobrepase los elementales diagnósticos y orientaciones reguladoras para el futuro de la acción educativa la cual debe funcionar de manera óptima teniendo como referente la supervisión educativa.

Los sistemas educativos son considerados como organismos vivos, y por lo tanto, supeditados al cambio desde los procesos y las instituciones y en estos escenarios, está presente la labor transformadora de la supervisión escolar. El mundo contemporáneo está en continuo y acelerado cambio debido a los avances científicos y tecnológicos y su consecuente implicación en las organizaciones sociales, en los sistemas políticos y por consiguiente, en los sistemas escolares. El mundo es una pequeña aldea global en distancias y tiempos más cortos a partir de los intercambios mercantiles, de capital, personales y de información. Igualmente, surgen nuevas lecturas sobre la justicia, la libertad, los sistemas democráticos, la diversidad cultural y la cohesión social.

Esta realidad no obvia las deudas sociales que tienen algunos países con la población infantil y juvenil, las cuales se evidencian en la desigualdad social convirtiéndose en freno para el desarrollo y la calidad educativa. Es entonces donde el papel de la supervisión educativa se convierte en un elemento transformador y aportante a la solución de los problemas emergentes. 


\section{Las funciones y finalidades de la Supervisión Educativa en la sociedad actual frente a los retos de la innovación y la calidad educativa en América Latina.}

La supervisión educativa en Iberoamérica es similar en sus funciones de informar, asesorar, mediar, controlar y evaluar. La labor de la supervisión es el puente entre la administración y el centro docente en casos como la aplicación de las normas entre otras funciones como las del proceso sistémico, e innovaciones administrativas, de tal forma que garantiza el ajuste del sistema educativo y su aplicación rápida y apropiada. Para Gil de Zárate (1855, p. 301), los supervisores son fundamentales para la instrucción pública dado que cumplen con una serie de funciones particulares. Una primera es la de información actualizada que tiene del sistema educativo en cuanto a centros educativos, normatividad, técnicas pedagógicas, familias, profesorado, administrativos, alumnado y demás personal relacionante.

Otra función es la de asesorar centros educativos, docentes, administrativos y directivos, dado su perfil profesional tanto en formación teórica y en el ejercicio práctico de la docencia. Su tarea está en la asesoría de procesos organizacionales, administrativos, de gestión y evaluación entre otras. También está la función de mediación en cuanto puente aproximativo entre la escuela y la administración sobre todo en contextos de mediación de conflictos escolares. En este orden, está la función de control, en tanto que tiene como referente comprobar, verificar y confirmar la aplicación de las normativas legales en el escenario de las instituciones escolares.

Como una derivada de esta función de control, está la de evaluación, la cual define la identidad del supervisor por cuanto valora constantemente el sistema educativo permitiendo reforzar lo que marcha bien, y superar lo que necesite ser intervenido para optimizar ambientes educativos, de tal forma que se está en una continua evaluación de lo formal a lo real de la institución escolar. Aun cuando debe el supervisor cumple esta función evaluativa el sistema educativo, es claro que ha de mantenerse distante como par evaluador externo y apoyando las evaluaciones internas institucionales.

Las funciones de evaluar y controlar conllevan mejorar el sistema evaluativo en aras de su calidad. Tal es el caso de la revisión, pertinencia de la aplicación, implementación de medidas de mejora, así como la interpretación de los resultados de las evaluaciones externas nacionales e internacionales. Igualmente, implica el seguimiento institucional para superar los eventos desfavorables e intervenir en función de la calidad total para el mejoramiento progresivo. (De Bono, 1993, p. 47). Es la evaluación, una cultura continua y progresiva enfocada hacia la calidad en el sistema educativo.

Fundamentalmente las finalidades de la supervisión se enmarcan en garantizar los derechos que tienen las personas a tener una educación de calidad y para mejorar la calidad educativa del sistema. En este sentido, los receptores más importantes son quienes se encuentran en edades de escolarización obligatoria. Para tal efecto, ha de evaluar desde lo formativo y continuo ajustando los cambios 
e innovaciones pertinentes y permanentes a partir de la evaluación institucional interna y externa respondiendo a los nuevos retos y características de la sociedad globalizada. Por lo tanto, el acompañamiento permanente ha de ser coherente y sistemático en toda la extensión de la formación de las personas, desde los más pequeños hasta la edad adulta. Este acompañamiento permite el perfeccionamiento de los procesos educativos de enseñanza aprendizaje para mejores resultados. El ideal de la evaluación, es que cobije a la totalidad de la comunidad como sistema educativo, y no solamente a los estudiantes.

El devenir histórico de la sociedad actual está matizado por movimientos evolutivos acelerados y más en lo relativo a los sistemas educativos y sus referentes como el ambiente del aula de clase, la estructura administrativa, los avances vertiginosos en el conocimiento, la incorporación de las tecnologías de la información y a comunicación, los paradigmas de la sociedad de la globalización, los nuevos modelos de movilidad de las personas en el horizonte mundial, los pluralismos ideológicos y multiculturales que afectan tanto a los colectivos sociales como a los individuos en sus singularidades personales. El hombre se enfrenta hoy a nuevas incertidumbres, a nuevas aventuras, Morin (1999, p. 46), y la ambivalencia de los valores.

En la escuela se proyecta la sociedad en la que está inmersa. En este sentido, se proyectan sus necesidades y sus contextos vivenciales que inciden en la autonomía de la escuela. En este sentido, el supervisor apoya en el control de las instituciones en medio de la diversidad de sus expresiones contextualizadas respondiendo a las necesidades de los centros educativos con idoneidad y calidad.

El servicio de inspección responde a la calidad de la educación y, por lo tanto, su función se direcciona hacia el mejoramiento de la práctica docente y el funcionamiento de los centros y los procesos de renovación educativa. Igualmente debe cuidar el cumplimiento de las normas correspondientes al sistema educativo, así como asesorar e informar a los diferentes sectores de la comunidad sobre sus derechos respecto al cumplimiento de sus deberes y obligaciones. No se trata de funciones de poder administrativo sino de apoyo en la consecución de la calidad y equidad educativa en todos los elementos y aspectos del sistema educativo y los procesos de enseñanza aprendizaje.

Los supervisores educativos son conocedores de la normatividad actual del sistema educativo, conocen la realidad en la que está inmersa la institución educativa, saben direccionar su función gestionadora en cuanto a las variables técnicas y pedagógicas. Así como conocen las normas, igualmente cuidan y controlan que se cumplan. Por otra parte, los supervisores realizan el seguimiento oportuno a los proyectos escolares, al desarrollo curricular y a la organización del centro educativo. Es clave también el apoyo que brindan a las instituciones escolares en el favorecimiento y optimización de los recursos humanos, materiales y funcionales. Igualmente cumplen con la tarea de asesorar a los docentes y directivos de la institución escolar, así como informan a la comunidad escolar sobre el devenir de la 
escuela, mediando entre ésta y la administración y velando por que se cumplan los estándares evaluativos.

Ahora bien, para que se pueda cumplir con estos indicadores evaluativos y de apoyo a la gestión escolar, es evidente y oportuno que los supervisores sean personas idóneas, muy bien preparadas y no como se les ha considerado como solamente controladores y sancionadores llevando a infravalorar y desvirtuar su valor intrínseco y fundamental. Es de por sí, una función necesaria y central para el sistema educativo y por tal razón, su perfil ha de responder a estos requerimientos profesionales y laborales, con amplio reconocimiento de las nuevas tecnologías de la información y la comunicación y de la administración educativa apoyándose en escenarios globales y en modelos de supervisión según su pertinencia significativa y aplicabilidad local.

La presencia de los supervisores en las instituciones educativas es necesaria para optimizar los procesos de calidad y equidad educativa en armonía con la dirección administrativa de los centros educativos y su funcionamiento sistémico. Para tal efecto, el eje direccionador es la cultura del cambio dejando de hacer las cosas de siempre por otras, la reforma en cuanto que se busca reformar, corregir y mejorar lo que ha estado funcionando mal, así como innovar, introduciendo nuevos horizontes aportativos.

Cambiar y reformar puede generar transformaciones radicales al sistema, sin embargo, es importante tener presente que muchos cambios pueden ser nefastos al sistema. En este sentido, la innovación responde a los retos emergentes a los que está abierta la institución escolar para no quedar relegada a los tiempos modernos los cuales avanzan a pasos agigantados.

El supervisor conoce el currículo y los elementos innovadores que se le pueden adecuar según los contextos educativos y sociales. La innovación implica "la incorporación de algo nuevo dentro de una realidad existente, en cuya virtud ésta resulta modificada". (Rivas, 2000, p. 20).

La innovación implica la creatividad en todos los escenarios escolares especialmente en las aulas, en los objetivos o propósitos y su aplicabilidad según los tiempos oportunos, en las competencias para la vida y la educación, en los contenidos curriculares, en la transversalidad y su incidencia en el currículo, en los modelos aplicativos a las actividades, en los materiales didácticos, en los recursos tecnológicos de la información y la comunicación, en los procedimientos de evaluación y metodologías de trabajo asumidas, en los modelos de inclusión así como la aceptación de áreas curriculares innovadoras. Todo esto debe llevar a comprender y estar atento a plantear una escuela que responda a paradigmas de nuevos y actualizados intereses sociales y sea atrayente a toda la comunidad escolar

La evaluación ha de ser rigurosa para que los procesos de mejoramiento implementados sean positivos. Estos cambios innovadores deben tener acompañamiento y seguimiento de tal forma que garanticen su viabilidad y esta tarea ha de cumplirla el supervisor a partir de la propuesta de cambio, de reforma 
y de innovación, una tarea que se ejecuta en equipo con otros profesionales de la educación y la administración

\title{
Calidad y enfoques de la supervisión.
}

La educación se direcciona a que el hombre construya pensamiento crítico y aprenda a coexitir en ambientes de sociabilidad buscando transformar la sociedad y el mejoramiento de las necesidades y la calidad en todos los procesos de control, supervisión y evaluación que exijan los entes administrativos como los Ministerios de Educación, Cultura y Deportes.

Ahora bien, esta supervisión educativa ha de estar ajustada según las directrices de la Constitución Política y demás normatividades del contexto educativo de tal forma que atiendan la integralidad de su quehacer en los Distritos, los sectores y los Planteles Escolares. Los Distritos escolares tienen áreas geográficas propias de su competencia, dadas sus funciones las cuales se ejecutan en planteles, centros, comunidades educativas, cátedras y servicios. Estas funciones, según Mogollón son las de:

\begin{abstract}
dirigir, coordinar, supervisary evaluar la ejecución de Políticas Educativas en el ámbito de su jurisdicción. Elaborar, ejecutar y controlar los programas de supervisión de la labor docente y administrativa que se realiza en los planteles. - Propiciar el mejoramiento de la calidad de la enseñanza. A Asesorar sobre los problemas Técnicos, Docentes y Administrativos que se presentan en los planteles. $-S$ uministrar orientaciones de carácter pedagógico, metodológico, técnico, administrativo y legal al personal en servicio. (p. 4).
\end{abstract}

Según el Ministerio de Educación (1987. P. 57), hay funciones que debe cumplir el Sector Escolar integrada por niveles de educación Preescolar, Básica, Media Diversificada, Especial, adultos y demás organismos educativos. Estas funciones se enfocan hacia la instrumentalización, supervisión, control y evaluación del desarrollo de la acción educativa, la asesoría a los directores para la solución de problemas, la organización de actividades para beneficiar la labor de los docentes, la orientación y asesoramiento de la organización, así como la atención a los padres y representantes.

La labor de supervisión del Distrito y el Sector Escolar, se hacen en compañía del director y los directivos en el plantel educativo lo cual redunda en la labor del docente y el alcance de los objetivos académicos y administrativos institucionales. Igualmente se debe involucrar el currículo escolar, el rendimiento de los estudiantes, la organización escolar, administrativa, así como los procesos de perfeccionamiento de los docentes determinando la eficiencia, efectividad y competencia del área educativa.

Ahora bien, para que se cumplan estas funciones, es importante que el supervisor conozca los procesos administrativos y académicos y de esta manera, según Sergiovanni (1971, p. 15), "provea un esfuerzo sistemático e integrado hacia el mejoramiento de la efectividad supervisora" para el fortalecimiento de las instituciones educativas y sus consiguiente beneficios y utilidades. Igualmente, la función supervisora revisa las necesidades, experiencias y recursos investigativos, didácticos, 
audiovisuales entre otros con los que cuenta la institución para su funcionamiento adecuado en orden técnico, organización administrativa y políticas educativas, Teixidó (1997:129-130), direccionadas hacia la calidad del sistema educativo, que en últimas es hacer mejor las cosas, Bounds y otro (1999:4), con orden, clarificando los roles correspondientes a las jerarquías y las acciones gerenciales basadas en las relaciones humanas interpersonales de la empatía, en saber escuchar, comunicar, confiar, delegar y orientar en el desarrollo de habilidades y técnicas para la enseñanza.

El supervisor ha de cultivar cualidades y condiciones personales, técnicas y académicas definidas a partir del reconocimiento de sí mismo, el autodominio, el liderazgo, la empatía, la organización, la creatividad, la solidez en su formación profesional pedagógica, metodológica, el manejo de las técnicas grupales para involucrar de manera participativa a los miembros de la comunidad educativa, el manejo de referentes de las tecnologías de la información y la comunicación, el manejo acertado de recursos técnicos, administrativos, bibliográficos y de relaciones humanas.

Para la Supervisión Educativa Nacional según el Programa de Reorganización y Descentralización (1997) la "Supervisión es el sistema a través del cual se desarrollan acciones en las distintas estancias territoriales para contribuir con el mejoramiento de la calidad de la educación..." (p22), que, en otras palabras, se trata de la fundamentación de la labor del supervisor en cuanto revisa, aplica y evalúa la aplicación de las normatividades en aras de la calidad del sistema educativo.

Imídeo citado por Fermin (1980) afirma que "La supervisión puede sintetizarse como asistencia a las actividades docentes, de manera de darles coordinación, unidad y continuidad, para que la escuela alcance con mayor eficiencia sus objetivos" ( $\mathrm{p} 15)$. El supervisor trabaja en orden a la mejora de los procesos de enseñanza aprendizaje del sistema educativo institucionales los cuales involucran el liderazgo, la planificación, la calidad de la gestión y la satisfacción de las necesidades educativas integrales.

El supervisor es un líder generador de cambios en el clima laboral institucional inspirando confianza, controlando, focalizando soluciones, promoviendo el mejoramiento de la calidad de los procesos de enseñanza aprendizaje, generando acciones proactivas, creativas desde la participación activa de todos los integrantes de la comunidad educativa. Planificará ejercicios de diagnóstico desde el enfoque sistémico identificando problemas y necesidades para la generación de alternativas frente a la resolución de conflictos. La planificación se encamina hacia la calidad de la gestión académica y administrativa involucrando a toda la comunidad académica y las necesidades del medio teniendo como referente la eficiencia en la labor supervisora.

Para Lepeley (2001) la gestión de la calidad "requiere la implementación sistemática de efectivos procesos de apoyo para asegurar la efectividad..." (p.68), y la calidad educativa. Sin embargo, según Hernández G citado por Marcano (2001) "es muy común hablar del deterioro de la calidad de la educación que se imparte en las instituciones del sistema educativo nacional...” (p.213), y en este escenario es claro que converge el papel del 
supervisor en alianza con el Distrito y el Sector Escolar. Para Fermín (1980), Nérici (1975) y Hierro (1974) hay diferentes enfoques de acuerdo a la especificación de la labor supervisora como son la inspección autocrática, fiscalizadora, supervisión correctiva, de inspección liberal, constructiva, preventiva, democrática y creativa.

Para Fermín (1980), en la inspección autocrática, predomina el método fiscalizador respecto a las actividades, denotando una función coercitiva y castigadora. La inspección liberal es de carácter constructiva de corte irresponsable en su concepto fundamental, mientas que la supervisión democrática promueve el mejoramiento profesional a partir de las normas, la experimentación y la investigación.

Para Nérici (1975), la supervisión ha evolucionado desde lo fiscalizador como inspección escolar en aras al cumplimiento de las normas, la planta física, matrículas, vacaciones y demás actividades. La fase constructiva dirigida hacia la actuación de los docentes, la creativa dirigida hacia el perfeccionamiento de los procesos de enseñanza aprendizaje y la fase autocrática en la que la labor del supervisor es la de prever lo concerniente al funcionamiento de la institución escolar. En este sentido, está la fase democrática, apoyada en los referentes científicos, humanos, creativos e innovadores.

En este orden, Hierro (1974), hace referencia a la supervisión constructiva que identifica defectos y errores para corregirlos, la preventiva según los momentos y contextos, la constructiva, que revisa métodos, objetivos, programas para la corrección de problemas y la supervisión creativa la cual enfatiza en el estímulo personal orientada hacia el docente. Para Angulo (1999), está la supervisión de inspección para el control de la responsabilidad administrativa, el mejoramiento de la eficiencia y de estándares para la toma de decisiones, la tecnológica apoyada en el método científico a partir de las relaciones de causa efecto apoyados en el coaching, la clínica que se aleja de las prácticas amenazantes de la supervisión promoviendo la intelectualización y profesionalización de los docentes a partir de efectivos procesos de información y comunicación y la supervisión crítica la cual describe los hechos, revisa la información, confronta para determinar las causas y reconstruye las relaciones dirigidas al cambio según las necesidades del medio para su aplicación.

\section{La supervisión pedagógica pública. Análisis de la gestión del supervisor como promotor del cambio educativo y mejoramiento en las escuelas}

En cuanto a la supervisión y el contexto sociopolítico, es importante identificar el papel que juega el supervisor educativo y la escuela, a partir de la década de los ochenta y los cambios generados por la globalización, (Castells, 1998, p 29), entendiendo que el supervisor ejerce funciones administrativo financieras y otras, de carácter educativo. Ahora bien, los cambios materiales y culturales implican una reestructuración de la sociedad capitalista matizada fundamentalmente por los aportes de la ciencia y las tecnologías de la información y la comunicación, los cuales inciden en las políticas y las relaciones de poder de la economía mundial y los 
escenarios de la identidad colectiva e individual. El Estado pierde hegemonía desde los constructos sociales y su representatividad como garante del bien común. Ahora bien, el mercado se convierte en un nuevo concepto emergente de la democracia emanado de la crisis política y su capacidad de poder. (Touraine, 2000, p. 319).

Según Núñez, (1986), el Estado está marcado por una fuerte debilidad referida al reformismo educativo y su incidencia en el fenómeno histórico, dada la ineficacia frente a las pedagogías tradicionales y sus reformas educativas hasta 1970. Esta realidad se debe a la presencia de un Estado centralizado y su consecuente dependencia administrativa con el sector educativo. No sin embargo, se generaron modificaciones en el sistema escolar (Núñez, 1995), como es la descentralización educativa y el apoyo al sector privado. Es evidente que desde 1990, la políticas se dirigen al cambio, conservando lo estructural para mejorar la calidad, la eficiencia y la equidad educativa. En este sentido, y acudiendo a las voces latinoamericanas, los cambio se direccionan hacia la descentralización. (Tedesco, 2001, p. 57).

La relación establecida entre el Estado y la escuela hacen que se proponga un nuevo concepto, el de la autonomía escolar como condición asociada al poder de la escuela y sus aportes en las metodologías, los procedimientos y la administración de los procesos de enseñanza aprendizaje fruto de gestión responsable y por ende, el papel que cumple el supervisor es fundamental en la representación, orientación, mediación y porte de las políticas educativas aplicadas en las escuelas. (MINEDUC: División de Educación General, 2002). Para García-Huidobro (2001), el papel del supervisor está dado principalmente por su gestión en cuanto media los objetivos de la reforma educativa y su vinculación con los docentes desde la autonomía educativa. Esta situación permitió la libertad curricular y la entrega de recursos. Igualmente, según el Min educación, (2002), el trabajo del supervisor se orienta hacia el:

"diagnostico, evaluación y promoción de la autoevaluación de los establecimientos, y a partir de estos resultados, discernir los apoyos apropiados al establecimiento educacional (I); elaborar planes de acción coherentes con los planes definidos por los establecimientos que atiende (III); promover la responsabilidad y autonomía de los establecimientos educacionales (V)" (p.5).

Respecto a la supervisión y la calidad del sistema escolar, están supeditados sus cambios a la realidad sociopolítica y a la necesidad de control de los objetivos y la protección de la educación como bien público y sus consecuentes resultados educativos evidenciados en el mejoramiento del rendimiento de los alumnos. En este sentido, el supervisor es un técnico y asesor que vigila el cumplimiento de las metas institucionales y la evaluación del desempeño de los docentes y su relación con el currículo. Igualmente ayuda a la resolución de los problemas asociados a la práctica escolar y sus procesos de modernización y desarrollo social. Por lo tanto, para Brunner y Elaqcua, (2003, p. 9), se hace necesario generar procesos de modernización del sistema de inspección y ayuda a las escuelas saliendo de los parámetros burocráticos hacia escenarios evaluativos que involucren a toda la comunidad educativa. En síntesis se busca, desde el control, evaluación y 
asesoramiento, la autonomía responsable de la escuela y todos los actores que con ella se relacionen en aras del aseguramiento de la calidad educativa.

Otro elemento reflexivo, corresponde al rol de la supervisión educativa en un contexto social de aseguramiento de la calidad escolar a partir del control, la asesoría y la evaluación. En este orden las funciones de control tienen que ver con la fiscalización en cuanto al cumplimiento de las normas establecidas y vigentes, los programas relacionados con el mejoramiento ministerial, el aseguramiento y el monitoreo del cumplimento de los indicadores mínimos de calidad, sus resultados y metas institucionales. En cuanto a la asesoría, esta busca apoyar técnicamente lo relacionado con temas didácticos y organizacionales, el fomento de la autonomía institucional, el empoderamiento de los actores escolares a partir del desarrollo, la innovación pedagógica, los recursos, los servicios públicos solicitados, el establecimiento de redes de cooperación internas y externas a la escuela. Por último, la evaluación la cual pretende recopilar información para valorar y prescribir decisiones en cuanto a indicadores mínimos de calidad, sobre la responsabilidad de las escuelas evidenciados en sus resultados.

En cuanto a las demandas del sistema escolar sobre la supervisión educativa, desde la perspectiva del Estado, el supervisor es un gestionador que opera funciones de control especialmente de evaluación. El contexto de la supervisión vista desde el carácter sociopolítico se enmarca en los procesos de globalización, respondiendo al Estado, la sociedad civil y los mercados en tanto que se exigen resultados y cumplimiento de estándares. Por otra parte, el contexto cultural es asumido a partir de los aportes de la era del conocimiento que exige la gestión de competencias simbólicas como el liderazgo transformador, el empoderamiento y la acción expresada en redes de apoyo. Para tal efecto, se hace necesario revisar la supervisión del sistema en aras de la calidad y el fortalecimiento de los vínculos entre el Ministerio y la praxis de las escuelas.

Otro factor es el contexto escolar en donde la supervisión opera para intervenir las escuelas con bajos resultados, ineficiente autonomía institucional, condiciones afectantes para la educabilidad y con poco control interno. Igualmente está el contexto científico a partir de modelos de gestión escolar, la eficacia escolar y la calidad total. Existen también los desafíos de la supervisión donde los contextos exógenos presionan sobre el control de la calidad y la evaluación a las escuelas, a las directivas por su gestión optimizadora de resultados, a los docentes y los estándares de gestión institucional y desarrollo profesoral.

\section{Modelos contemporáneos sobre la supervisión escolar y su impacto en las reformas educativas correspondientes al proceso de transformación de los sistemas educativos en América Latina}

Para Braslavsky (2001), hay una compleja dinámica de los sistemas educativos en América Latina la cual parte de dos paradigmas o modelos; el comunal, que surge de abajo hacia arriba y el modelo estatalizador, que es inverso, es decir, de arriba hacia abajo. En América Latina, se buscó combinar ambos modelos dando 
especial énfasis a lo piramidal, jerárquico, centralista y autoritario en cuanto a lo administrativo y el poder decisorio lo cual no permite lo colaborativo, lo innovador, lo comunitario y participativo social donde la escuela es el eje articulador de lo transformador educativo. La reforma de la educación, por lo tanto, hace referencia a la transformación del currículo y la renovación de los enfoques pedagógicos direccionada a los procesos de enseñanza aprendizaje. Por otra parte, la reforma de los sistemas educativos, alude a los cambios en la gestión institucional tanto macrosistémicos como mesoestructurales.

El mejoramiento de la calidad y equidad educativa no han sido gratuitas. (Martinic y Pardo, 2001; García-Huidobro, 1999, entre otros). La reforma educativa obedece a la creciente globalización y las transformaciones aceleradas del mercado internacional y las exigencias a los sistemas educativos y su aporte a la formación del capital humano y los objetivos sociales de justicia, libertad, democracia y cohesión social. Según Zorrilla y Barba, (2008), son diversos los factores políticos, económicos, jurídicos, técnicos, ambientales, culturales y psicosociales que motivan los cambios del sistema educativo. Latinoamérica experimenta estos cambios de manera significativa en su quehacer cotidiano. Por una parte se amplía la cobertura y el acceso a la escuela y por otra, se presenta de manera tangible, insuficiencias e inconsistencias para el desarrollo armónico del sistema educativo como garantía al derecho a la educación.

Los cambios generan sinergias que operan de manera complementaria e independiente. Estas sinergias son políticas, científicas, burocráticas, administrativas, técnicas, profesionales, prácticas y pedagógicas a partir de tiempos, saberes y personas como actores sociales, políticos y educativos entre quienes están los docentes, los directivos, los gobernantes, los padres de familia, las organizaciones así como sus intereses y comprensiones de la realidad. Para tal efecto, se hacen necesarios ambientes de colaboración y compromiso con sentido de diversidad en el diseño de estrategias renovadoras de intervención, (Carron, 1997), con sentido de coherencia frente a la interdependencia de factores en el diseño y la implementación de cambios teniendo como centro al docente y sentido de realismo, en cuanto se atiende a las escuelas en sus contextos circunstanciales y reales.

Un factor fundamental es la persona como agente educativo y en este orden, están los niños, las niñas y los jóvenes quienes en el ambiente escolar, construyen su proyecto de vida justa y convivencial. Todos son responsables desde sus ámbitos de competencia y atribución, así como desde sus niveles de autoridad y organización escolar en relación con el aula y la escuela. La escuela gestiona con eficiencia y eficacia en el logro de sus objetivos y la toma de decisiones en el orden administrativo, curricular, organizativo y pedagógico.

La educación es un derecho ejercido por todos en aras de la calidad y la transformación escolar la cual es apoyada por la supervisión, (Calvo et al., 2002), desde su posición estratégica mesoestructural al vincular la autoridad educativa superior con los centros escolares. En este sentido, se refuerza la función del supervisor en cuando cuidado, vigilancia y apoyo de la organización escolar. Pasa 
del control fiscalización al control apoyo del desarrollo curricular, la capacitación y actualización docente, la evaluación, el diseño y operación de propuestas de mejora. Es una función vinculativa y articuladora entre las políticas educativas y la operacionalización cotidiana en el entorno escolar. La educación ha sido asumida como función pública técnica para asegurar el progreso de la escuela. (Puelles, 1990). La vigilancia de las escuelas era tarea de los padres de familia y comisiones escolares que luego es asumida por inspectores escolares como fiscalizadores, burócratas controladores y sancionadores frente a la norma laboral y administrativa. Tenían el poder de control oficial y político, de subordinación y movilización docente, de manejo de recursos financieros y mecanismos de control. A partir de algunos eventos educativos internacionales, se ha considerado la educación como fundamental para el desarrollo de los pueblos y en este sentido, el conocimiento y el aprendizaje son ejes transformadores productivos.

Los nuevos ciudadanos se forman con calidad y equidad, con calidad en la enseñanza y el servicio desde la organización y las dinámicas legales y administrativas fruto de la gestión. (Ezpeleta, 1995: 1). Se considera que los supervisores son actores clave y estratégicos para iniciar acciones que impliquen reformas del sistema educativo. La atención se ha centrado en su función disyuntiva entre lo pedagógico y lo administrativo desde los contextos teóricos y prácticos de la realidad educativa con la rendición de cuentas como resultados educacionales y la función de control de apoyo teniendo como referentes el monitoreo, la animación, el seguimiento y la evaluación.

Un referente es el Estado de Aguascalientes del centro de México. En la década de los noventa se enfatizó en la descentralización de la educación por parte del gobierno de tal manera que los estados deberían asumir la responsabilidad y los recursos para accionar en todos los niveles educativos y en la formación inicial y continua de los docentes. (Zorrilla, 1998). Se trata de una significativa y profunda reforma curricular y pedagógica según el contexto regional planteado en la que primaría la calidad y equidad, la cobertura y acceso, el aseguramiento de la permanencia así como la identificación de las singularidades y necesidades de algunas escuelas en particular. La reforma implicó que los supervisores, los directivos y los maestros asumieran los problemas y plantearan soluciones. El supervisor por lo tanto, asume responsabilidades institucionales y profesionales, como puente entre la administración y las escuelas, y la necesidad de prepararse con idoneidad para asumir su cargo.

Existía una relación intrínseca entre las políticas educativas del centro del país y la labor multiplicadora del supervisor a partir de la formulación de los objetivos que se pretendían lograr y la puesta en escena de procesos de negociación entre los distintos actores políticos y su influencia partidista. Igualmente su función se direcciona a la priorización de los recursos financieros para el gasto educativo estatal. Otro factor funcional tiene que ver con la regulación de la normatividad y sus consecuentes responsables para ejecutarla en medio de las circunstancias burocráticas, de resistencias, de capacitación y coordinación entre los distintos liderazgos y grupos de trabajo. En el caso concreto de Aguascalientes, la reforma 
favoreció la distribución del poder, la autoridad y la responsabilidad. Igualmente favoreció la cercanía de todos los agentes de la comunidad educativa dando lugar al trabajo en equipo y colegiado más allá de la tradicional función individual del supervisor. Se articularon territorialmente las zonas escolares dentro de los contextos municipales así como se logró la conciliación de lo administrativo con lo pedagógico en las realidades escolares concretas desde sus diagnósticos, evaluación y planeación correspondientes al actuar del supervisor.

Reformar implica hacer un ejercicio gradual para involucrar a todos los actores a partir de su estabilidad relativa de los supervisores, el monitoreo frecuente de la experiencia y los procesos de capacitación del personal, actualización, cambio e innovación de las tareas cotidianas del ejercicio supervisor educativo. Esta reforma mantuvo de manera equilibrada los poderes políticos y sindicales desde la mirada política, y desde la mirada académica, se mantuvo la formación profesional y humana de los supervisores aun cuando no incidiera profundamente en las prácticas escolares. La consolidación de la reforma exige el acceso y permanencia de los supervisores en su condición laboral, su formación profesional específica y su capacidad de toma de decisiones autónomas desde los contextos administrativos y laborales. Por otra parte, se hace necesario definir la injerencia del ámbito central de la educación básica, dadas sus consecuencias presupuestales y normativas, cosa que le corresponde al gobierno estatal.

\section{Conclusiones}

La supervisión educativa en Latinoamérica es parecida en sus funciones correspondientes a informar, asesorar, mediar, controlar y evaluar. En otras palabras, no se ha encontrado diferencias en su actuar. Por otra parte, las finalidades de la supervisión se enmarcan en garantizar los derechos que tienen las personas a tener una educación de calidad y para mejorar la calidad educativa del sistema. El devenir histórico de la sociedad actual está matizado por movimientos evolutivos acelerados y más en lo relativo a los sistemas educativos a los cuales el supervisor educativo ser ve abocado a enfrentar para brindar la ayuda pertinente a las instituciones.

El servicio de inspección responde a la calidad de la educación y, por lo tanto, su función se direcciona hacia el mejoramiento de la práctica docente y el funcionamiento de los centros y los procesos de renovación educativa. La presencia de los supervisores en las instituciones educativas es necesaria para optimizar los procesos de calidad y equidad educativa en armonía con la dirección administrativa de los centros educativos y su funcionamiento sistémico.

El papel que cumple el supervisor es de vital importancia en la construcción de ambientes educativos de calidad atendiendo a los nuevos retos innovadores que plantean las sociedades de las tecnologías de la información y la comunicación en el gran escenario de la globalización que toca todos los horizontes posibles de los cuales no escapa el universo de los pueblos latinoamericanos.

La labor de la supervisión educativa ha de ser prudente a la hora de tener en cuenta los diversos enfoques que se establecen para asegurar la calidad educativa 
de los procesos de enseñanza aprendizaje. La realidad educativa es cambiante y a partir del liderazgo ejercido por el supervisor, se pueden planear transformaciones profundas en el sistema educativo.

El supervisor educativo ha de ser un profesional idóneo con dominio de los escenarios y contextos académicos, técnicos y administrativos de la realidad educativa apoyando la realización del ser a partir de la vivencia de valores donde la persona es el centro de la labor educativa. La labor del supervisor busca ser más democrática en su quehacer generando ambientes democráticos de participación involucrando a todos los miembros de la comunidad educativa como directivos, docentes, estudiantes, padres de familia desde el acompañamiento pedagógico frente a las nuevas exigencias de la sociedad y de las instancias gubernamentales como los Ministerios de educación.

Es evidente que este tipo de reflexiones y análisis conlleva cuestionarse sobre los cambios efectivos educativos en aras de la calidad educativa y el consecuente desarrollo organizacional y la implementación de estándares que propendan por la calidad en las escuelas y más aún en escuelas que demuestran bajos rendimientos ancladas en las burocracias tradicionales.

Debatir sobre la autonomía escolar en el contexto latinoamericano no ha sido una fortaleza y por lo tanto su incidencia se da en el quehacer del supervisor. Según las directrices del Estado, la autonomía y responsabilidad escolar se ha de ajustar a los parámetros estratégicos del ministerio y su currículo oficial de resultados. No se trata de debates planteados a partir de principios sobre la autonomía sino también desde las variables técnicas, las funciones del supervisor, la incidencia del medio externo estatal así como la interna escolar.

América Latina no se aparta de los modelos internacionales de estandarización para medir la calidad de los rendimientos escolares lo cual le da para obtener respuestas formales de la escuela. Los estándares e indicadores son guías para el mejoramiento de la calidad pero no son simples fines dado que los cambios son complejos. Para Hargreaves (2003), el cambio educativo no está supeditado a eventos exógenos o endógenos, sino a las metodologías y el uso de las competencias simbólicas adecuadas desde el liderazgo del supervisor para el empoderamiento de los actores escolares, su autonomía, los altos estándares de desarrollo organizacional y apertura al aprendizaje.

Según las necesidades locales, la transformación de la supervisión se hace efectiva porque es un auténtico mecanismo de apoyo y mejoramiento del entorno escolar en calidad y equidad para las niñas, niños y adolescentes. La reforma ha brindado avances relativos tanto para docentes como para los supervisores en cuanto a su capacitación profesional. A un supervisor, según Tapia, (2004), se le ha de reconocer su significativa experiencia tanto personal como institucional en su ejercicio laboral individual y colectivo. 


\section{Referencias}

Angulo, F., (1999) La supervisión Docente, Dimensiones, Tendencias y Modelos. España: Ed. Akal Textos.

Bounds, G. y Otro. (1999) Supervisión. México: Internacional Thomson Editores.

Braslavsky, C. (2001). La dinámica del cambio educativo para el siglo xxi. Acerca de la emergencia del paradigma en red. Buenos: AIRES-IIPE-UNESCO.

Braslavsky, C. y Cosse, G. (1996). Las actuales reformas educativas en América Latina: cuatro actores, tres lógicas y ocho tensiones. Documento no 15 . Santiago de Chile: Programa de Promoción de la Reforma Educativa en América Latina.

Brunner, J. J., Elaqcua, G. (2003). Informe Capital Humano en Chile. Universidad Adolfo Ibáñez.

Calvo, B.; Zorrilla, M.; Tapia, G. y Conde, S. (2002). La supervisión escolar de la educación primaria en México: prácticas, desafíos y reformas. París: Instituto Internacional de Planeamiento de la Educación-UNESCO.

Carron, G. (1997). Mejorar la calidad de la escuela primaria. Resultados de una investigación internacional. Abril. París: IIEP/Prg.GC/97.161.

Castells, M (1988) La era de la información: Economía, Sociedad y Cultura. "La Sociedad en Red". Volumen No 1, Madrid, España: Editorial Alianza

De Bono, E. (1993). El pensamiento lateral. Manual de creatividad. Barcelona: Paidós Ibérica.

Ezpeleta, J. (1995). Gestionar la calidad educativa: el cambio en la supervisión, proyecto de investigación presentado y aprobado por el Sistema Regional de Investigación Miguel Hidalgo-conacyt. [Mecanograma].

Fermín, M. (1980) Tecnología de la Supervisión Docente. Buenos Aires: Editorial Kapelusz, S.A.

García-Huidobro, J. (ed.) (1999). La reforma educacional chilena. Madrid: Editorial Popular.

García-Huidobro, J.E. (2001) Conflictos y alianzas en las reformas educativas. Siete tesis basadas en la experiencia chilena, En Martinic-Pardo (Ed.). Economía Política de las Reformas Educativas en América Latina, CIDE, PREAL, pp. 205-218.

Gil de Zárate, A., (1855), De la instrucció pública en España, Oviedo: Pentafla.

Hargreaves, Andy (2003). Enseñar en la sociedad del conocimiento (La educación en la era de la inventiva). Barcelona: Octaedro. 
Hierro, L., (1974) Enfoque Práctico de la Supervisión Escolar. Buenos Aires: Editorial Kapelusz.

Lepeley, M., (2001) Gestión y Calidad en Educación. Un modelo de Evaluación. LTDA. Chile: Editorial Mac-Graw/ Interamericana de Chile.

Marcano, A., (2001) Acuerdo semántico en la Comunicación, aplicada al proceso de Enseñanza y Aprendizaje en la Mención de Educación Comercial de la Facultad de Ciencias de la Educación de la Universidad de Carabobo. Valencia: Revista Ciencias de la Educación. Año 1 No 17.

Martinic, S. y Pardo, M. (eds.) (2001). Economía política de las reformas educativas en América Latina. Santiago de Chile: CIDE.

MINEDUC, División de Educación General (2002). Criterios de política para la supervisión técnico pedagógica período 2002-2005, Jornada Nacional de Gestores de la Supervisión 19, 20 y 21 de agosto 2002.

Ministerio de Educación, (1987). Manual de Organización de las Zonas Educativas. Caracas. Venezuela: Dirección de Información y Relaciones Públicas.

Ministerio de Educación, (1997). Programa de Reorganización y Descentralización Caracas. Venezuela: FEDUPEL.

Morin, E. (1999). Los siete saberes necesarios para la educación del futuro. París: UNESCO.

Nérici, I., (1975) Introducción a la Supervisión Escolar. Buenos Aires: Editorial Kapelusz.

Núñez, Iván (1986). Experiencia de cambio educativo durante el Estado de compromiso. PIIE, Informe de Investigación, Cap. XIII, Santiago de Chile.

Núñez, Iván (1995). Hacia un nuevo paradigma de reformas educativas: la experiencia chilena. Revista Pensamiento Educativo, Facultad de Educación, PUC, Vol. 17.

OCDE (2004). Revisión de Políticas Nacionales de Educación: Chile. Santiago: Centro para la cooperación con los países no miembros de la OCDE, Chile.

Puelles, M. (1990). "Revolución Francesa y Educación: su incidencia en la génesis del sistema educativo español”. En: Ossembach, G. y Puelles, M. La Revolución Francesa y su influencia en la educación española. Madrid: UNED y Universidad Complutense de Madrid, 65-102.

Rivas Navarro, M. (2000). Innovación educativa. Teoría, procesos y estrategias. Madrid: Síntesis.

Sergiovanni, T., (1971) Emerging Patterns of Supervisión. Human. Perspectives. New York: Mc Graw-Hill. 
Tapia, G. (2004). "La supervisión escolar. Perspectivas de reforma e innovación en el marco de la reforma educativa mexicana”. Tesis de Maestría en Innovación Educativa. Universidad de Guanajuato-Instituto de Investigaciones en Educación.

Tedesco, Juan Carlos (2001). Desafíos políticos de las reformas de la educación. En Martinic-Pardo (Ed.). Economía Política de las Reformas Educativas en América Latina, CIDE, PREAL, Santiago, pp. 53-61.

Teixidó, M., (1997) Supervisión del Sistema Educativo. Barcelona: Editorial Ariel, S.A.

Touraine, Alain (2000) Crítica de la Modernidad. México: Fondo de Cultura Económica.

Zorrilla, M. y Barba, B. (2008). "Reforma educativa en México: descentralización y nuevos actores”, Sinéctica. Revista electrónica.

Zorrilla M. (1999). "De un modelo armado a uno para armar. Descentralización e innovación educativas en Aguascalientes 1992-1998”. En: Pardo, María del Carmen (coord.). Federalización e innovación educativa en México. México: El Colegio de México-Centro de Estudios Internacionales, 301-398.

Zorrilla, M. (1998). "Federalización, supervisión escolar y gestión de la calidad de la educación”. En: Latapí, Pablo (coord.). Un siglo de educación en México. Tomo I, cap. IX. Biblioteca Mexicana. México: FCE, 319-357. 